\title{
Equipamento triaxial cíclico de grande escala para análise mecânica de lastro ferroviário
}

\author{
Antonio Merheb¹, Rosângela Motta², Liedi Bernucci³, Edson Moura4, \\ Robson Costa ${ }^{5}$, Tiago Vieira ${ }^{6}$, Fernando Sgavioli ${ }^{7}$
}

\begin{abstract}
Resumo: O lastro é a camada responsável por dar suporte à superestrutura ferroviária, sendo carregada e descarregada repetidamente pela passagem dos trens. Em vista disso, a compreensão das características de tensão-deformação deste material granular que é utilizado nesta camada é importante para a otimização das operações de manutenção, garantindo um transporte eficiente e seguro. O material utilizado em camada de lastro ferroviário é normalmente constituído de frações granulométricas graúdas, com gradução uniforme, dificultando a execução de testes de grande escala em laboratório. 0 objetivo deste artigo é avaliar a aplicabilidade de um novo arranjo experimental empregando prensa hidráulica para ensaio triaxial de carga repetida, de grande escala de laboratório (com diâmetro de $400 \mathrm{~mm} \times$ altura de $800 \mathrm{~mm}$ ), que permite testar partículas de lastro em dimensões reais de campo, sem a necessidade de escalonamento e garantindo maior representatividade. Assim, é possível simular o carregamento que ocorre com a passagem de trens, a fim de se observar o comportamento do lastro quanto a deformações resilientes e permanentes. Um ensaio triaxial cíclico realizado com tal equipamento mostrou a viabilidade de preparação de corpo de prova em tais dimensões e de obtenção de resultados de módulo de resiliência e deformação permanente compatíveis com o esperado.
\end{abstract}

Palavras-chave: Lastro ferroviário. Ensaio triaxial cíclico.

\begin{abstract}
The ballast layer usually is responsible for giving support to the railway superstructure and is loaded and unloaded repeatedly due to the passage of trains. In this context, the comprehension of the stress-strain characteristics of this noncohesive material used in that layer is important for optimizing maintenance operations, thus ensuring a safe and efficient transport. The material used in ballast layer is usually composed of highly coarse-graded gradation, hence large-scale laboratory tests are more difficult to conduct. The purpose of this paper is to evaluate the applicability of a new Brazilian largescale triaxial apparatus $(400 \mathrm{~mm} \times 800 \mathrm{~mm})$ that allows testing large-size particles such as in the field, without the need of scaling aggregates and ensuring more representativeness. Thus, it is possible to simulate loading that occurs due to the passage of trains, in order to observe the mechanical behavior of ballast in terms of resilient and permanent deformations. A cyclic triaxial test carried out with that apparatus showed the availability of preparing large-size samples and obtaining results of resilient modulus and permanent deformation compatible with what was being expected.
\end{abstract}

Keywords: Railroad ballast. Cyclic triaxial test.

\section{INTRODUÇÃO}

O lastro ferroviário é a camada responsável por dar suporte à superestrutura das vias férreas ou via permanente, composta pelos trilhos, dormentes e fixações, sendo carregada e descarregada repetidamente pela passagem dos trens, e responsável pelo amortecimento de impactos e

1 Escola Politécnica da Universidade de São Paulo (antoniomerheb@usp.br).

2 Escola Politécnica da Universidade de São Paulo (rosangela.motta@usp.br).

3 Escola Politécnica da Universidade de São Paulo (liedi@usp.br).

4 Escola Politécnica da Universidade de São Paulo (edmoura@usp.br).

5 Escola Politécnica da Universidade de São Paulo (costa.robsonc@gmail.com).

6 Escola Politécnica da Universidade de São Paulo (tiagovr@gmail.com).

${ }^{6}$ VALE S.A. (fernando.sgavioli@vale.com).

Manuscrito recebido em 12/06/2014 e aprovado para publicação em 27/08/2014. Este artigo é parte de TRANSPORTES v 22, n. 3, 2014. ISSN: 2237-1346 (online).

DOI: http://dx.doi.org/10.14295/transportes.v22i3.804. redução de desgaste severo de trilhos. Em vista disso, a compreensão das características de tensão-deformação do material granular utilizado nesta camada, sujeito a carregamento repetido, é importante para conhecer os ciclos de vida destes materiais de modo a programar as operações de manutenção, garantindo um transporte eficiente e seguro.

O estudo do comportamento mecânico do lastro de ferrovias já foi realizado por diversos autores, tanto em campo quanto em laboratório. No âmbito laboratorial, o comportamento elasto-plástico de materiais granulares é geralmente investigado por meio de ensaios triaxiais, como apresentado por Alva-Hurtado et al. (1981), Stewart (1986); Diyaljee (1987); Indraratna et al. (1997) e Nalsund (2010). Segundo Indraratna et al. (1998), o equipamento de ensaio triaxial é um dos mais versáteis e úteis para a determinação das propriedades de resistência e 
deformação de materiais geotécnicos.

A aplicação da carga vertical é feita através de uma célula de carga e o confinamento de corpos de prova cilíndricos (envolvidos por uma membrana) é obtido por meio de um fluido (água, ar ou óleo) sob pressão. A Figura 1 mostra uma representação esquemática do equipamento convencional utilizado para ensaios triaxiais. Como a tensão de confinamento é aplicada por um fluido, a tensão principal intermediária $\left(\sigma_{2}\right)$ é igual à tensão principal menor $\left(\sigma_{3}\right)$, recebendo o nome de tensão confinante. A tensão principal maior $\left(\sigma_{1}\right)$ é então obtida pela soma da tensão confinante $\left(\sigma_{\mathrm{c}}\right)$ com a tensão imposta pelo pistão $\left(\sigma_{\mathrm{d}}\right)$. Uma representação destas tensões também é ilustrada na Figura 1.

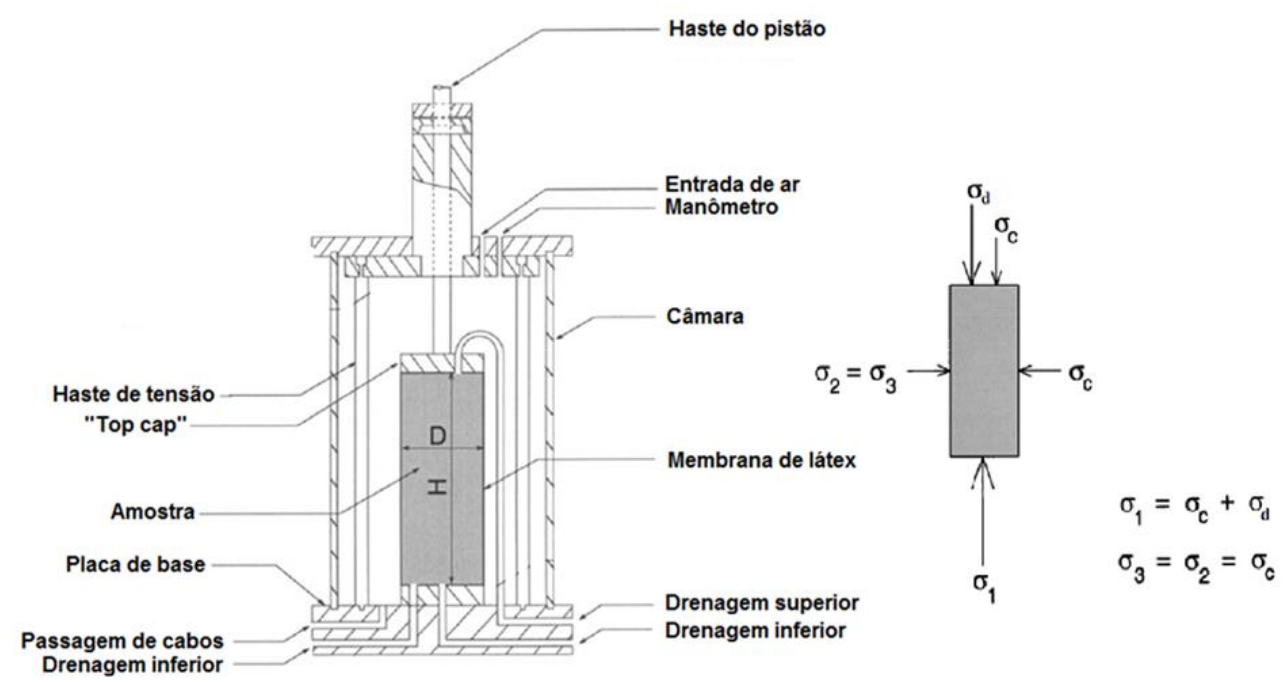

Figura 1 - Representação de equipamento de ensaio triaxial e estado de tensão assimétrico em um teste convencional (Suiker et al., 2005)

Para o estudo de lastros ferroviários em laboratório, a maior dificuldade encontrada é relativa à dimensão do material, cujos diâmetros máximos das partículas chegam a ser da ordem de 63,5 mm, impedindo a utilização do equipamento triaxial convencional que é usualmente empregado para solos e outros materiais granulares utilizados no meio rodoviário (menor dimensão). Outro fator que dificulta ensaios destes tipos e materiais é a manipulação de amostras de grandes dimensões, sendo necessário o uso de equipamentos não habituais nos laboratórios.

Para contornar este problema pode ser feita a translação da curva granulométrica para ensaiar o material em escala reduzida (decalada), porém ressalta-se que resultados divergentes entre amostras decaladas e de grandes dimensões foram evidenciadas por Indraratna et al. (1998) e Merheb (2014). Desta forma, a realização de ensaios em grande escala é mais realista, pois os corpos de prova com material de lastro são preparados com curvas granulométricas similares àquelas reais de campo e são submetidos a tensões características de ferrovias.

De acordo com Skoglund (2002), os valores típicos da relação $\mathrm{D} / \mathrm{d}_{\text {máx }}$ (onde: $\mathrm{D}=$ diâmetro do corpo de prova; $\mathrm{e} \mathrm{d}_{\text {máx }}=$ diâmetro máximo da partícula) variam de 5 a 7 , demonstrando que o diâmetro do corpo de prova deve ser considerável para que haja uma distribuição suficientemente representativa de partículas ao longo de sua dimensão. Este cuidado permite que o comportamento do conjunto de partículas seja testado como um todo, e não de um grupo reduzido, até não representativo, do conjunto integral.

Outros autores como, por exemplo, Suiker et al. (2005) e Sevi (2008) trabalharam com ensaios em grande escala no âmbito ferroviário e recomendam que o diâmetro da maior partícula de agregado seja menor que $1 / 6$ do diâmetro do corpo de prova, para que os efeitos do equipamento possam ser desprezados. Tal relação também está descrita na norma ASTM D5311 (2004) para solos; para que as dimensões do corpo de prova sejam consideradas adequadas para ensaios de módulo de resiliência, a AASHTO T292-91 recomenda que $90 \%$ dos agregados tenham diâmetro máximo seis vezes menor do que o diâmetro dos corpos de prova, e que todos os agregados tenham diâmetro máximo inferior a um quarto do diâmetro do corpo de prova. Por outro lado, considerando a relação entre H/D (altura e diâmetro), Bishop e Green (1965) recomendam que a proporção seja de 2. 
Neste caso, os autores relatam que este valor é ideal para eliminar o efeito de atrito nas extremidades da amostra.

Em função das dimensões citadas, poucos são os laboratórios no mundo que conseguem realizar estes ensaios, porém não somente devido ao tamanho graúdo das partículas, mas também pela grande capacidade de carga necessária ao equipamento que impõe os carregamentos. Ensaios com lastros ferroviários, principalmente aqueles que serão submetidos a tráfego de trens carga, requerem equipamento de reação (atuador) com capacidade de carga elevada, visto que existem ferrovias que operam com carregamentos, por exemplo, que chegam a 40 toneladas/eixo na Austrália. Além da robustez necessária à prensa atuadora, é de fundamental importância que o equipamento esteja calibrado e execute a simulação dos ciclos de carregamento de forma precisa, pois sob elevadas frequências, diversos ensaios de grande escala já evidenciaram instabilidade (Aursudkij, 2007; Fortunato, 2005).

A Tabela 1 mostra alguns locais onde foram realizados estudos de lastros por meio de ensaios triaxiais de grande escala. Cabe mencionar ainda uma pesquisa nacional em que Malysz (2009) desenvolveu equipamento traiaxial de grande porte $(250 \mathrm{~mm}$ x $500 \mathrm{~mm}) \mathrm{com}$ o objetivo de avaliar o comportamento mecânico de agregados utilizados como camada de pavimentos. Entretanto, o estudo do lastro ferroviário por meio de equipamento triaxial de grande escala é inexistente no país.

\begin{tabular}{|c|c|c|c|c|c|}
\hline Pesquisadores & Ano & $\begin{array}{l}\text { Diâmetro do } \\
\text { equipamento } \\
(\mathrm{mm})\end{array}$ & $\begin{array}{l}\text { Altura do } \\
\text { equipamento } \\
(\mathrm{mm})\end{array}$ & $\begin{array}{l}\text { Diâmetro máx. } \\
\text { da partícula } \\
(\mathrm{mm})\end{array}$ & Local \\
\hline Raymond, Diyaljee & 1979 & 230 & 460 & 38 & Canadá \\
\hline Alva-Hurtado et al. & 1981 & 305 & 610 & 30 & Estados Unidos \\
\hline Suiker et al. & 2005 & 254 & 645 & 38 & Estados Unidos \\
\hline Sekine et al. & 2005 & 300 & 600 & 63,5 & Japão \\
\hline Lackenby et al. & 2007 & 300 & 600 & 53 & Austrália \\
\hline Anderson, Fair & 2008 & 236 & 455 & 50 & Inglaterra \\
\hline Sevi et al. & 2009 & 419 & 864 & 63,5 & Estados Unidos \\
\hline Aursudkij et al. & 2009 & 300 & 450 & 50 & Inglaterra \\
\hline Nalsund & 2010 & 300 & 600 & 63 & Noruega \\
\hline Ebrahimi et al. & 2010 & 305 & 610 & 60 & Estados Unidos \\
\hline
\end{tabular}

Atualmente ainda não existem ensaios triaxiais normatizados para material de lastro, referentes a módulo de resiliência e deformação permanente, o que leva à uma adaptação do ensaio triaxial empregado para solos granulares, em função da necessidade do pesquisador. Em geral, varia-se a tensão de confinamento $\left(\sigma_{c}\right)$ e a tensão-desvio $\left(\sigma_{\mathrm{d}}\right)$ para ensaios de módulo de resiliência, enquanto estas tensões não variam ou variam em poucas combinações no estudo de deformação permanente, cujos resultados são usualmente expressos em função do número de aplicações de carga.

\section{EQUIPAMENTO}

Um equipamento triaxial capaz de ensaiar partículas de grande dimensão foi desenvolvido com capacidade para testar corpos de prova de $400 \mathrm{~mm}$ de diâmetro e $800 \mathrm{~mm}$ de altura, tendo sido concebido para realizar testes cíclicos em diferentes pressões confinantes, frequências, formas de pulso e condições de drenagem. Alguns detalhes importantes também fizeram parte 
da concepção do equipamento, como: (i) confecção de um molde em aço (pesando cerca de 235 $\mathrm{kg}$ ), composto de duas bases (superior e inferior) e quatro subdivisões laterais, que conferem robustez necessária ao conjunto e facilidade de desmoldagem. Ressalta-se que tanto a base inferior quanto à superior possuem orifícios que permitem a aplicação do confinamento à vácuo, diferentemente de triaxiais tradicionais em que a pressão confinante é fornecida por um fluido contido no interior da câmara triaxial. Este sistema de vácuo foi escolhido devido ao tamanho do corpo de prova, onde a adoção de uma abordagem semelhante a uma célula triaxial tradicional com fluido teria que ser demasiadamente robusta para os padrões do laboratório.

Dentro deste contexto, o sistema de vácuo é capaz de aplicar tensões confinantes de até $80 \mathrm{kPa}$, sendo este valor suficiente para o estudo de agregados empregados em ferrovias (Sevi, 2008); (ii) confecção de uma grua de transporte com sistema de içamento hidráulico para movimentação de amostras de grandes dimensões no laboratório, com capacidade para comportar um corpo de prova (molde + material) de até $600 \mathrm{~kg}$; e (iii) Aquisição de um aparelho elétrico de vibração, próprio para as dimensões do equipamento, para compactar o material no molde, em camadas, por vibração. Os detalhes de toda a aparelhagem podem ser vistos na Figura 2.

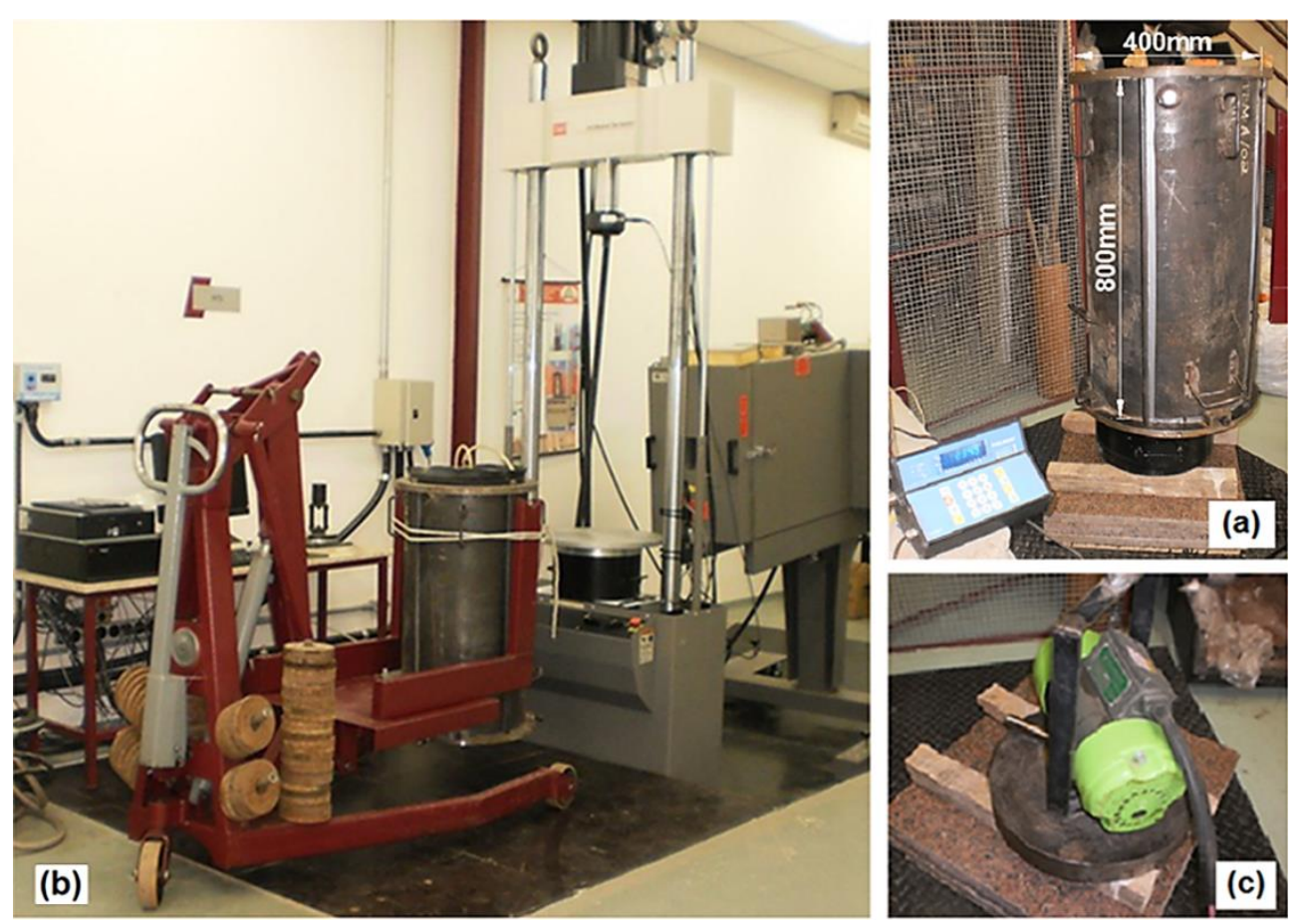

Figura 2 - Aparato triaxial de grande escala (a) pesagem do molde, (b) grua de transporte e bancada de ensaio, e (c) vibrador elétrico para a compactação

O carregamento é imposto pelo atuador Material Test System (MTS 810), que é capaz de aplicar cargas de até $25 \mathrm{kN}$. Nos testes iniciais para o desenvolvimento deste equipamento foi atingida uma frequência de ensaio de $9 \mathrm{~Hz}$ (que corresponde à velocidade de um trem de carga típico de 60 a $70 \mathrm{~km} / \mathrm{h}$ ), sem que houvesse nenhum desequilíbrio hidráulico (observou-se que em frequências superiores havia perda de harmonia entre as tensões solicitadas e as aplicadas, por conta da instabilidade hidráulica que um corpo de prova de grandes dimensões ocasiona quando submetido a tensões elevadas). De qualquer forma, foi atingida a frequência típica de carregamento para um trem de carga, que é da ordem de 8 a $10 \mathrm{~Hz}$ (segundo Aursudkij, 2007), tornando o ensaio que foi realizado como sendo representativo da situação para trens de carga.

Outro ponto fundamental para a realização do ensaio em grande escala é a colagem da membrana de látex que envolve o material. Pelo fato de não existir a comercialização de membranas com tamanhos personalizadas com $400 \mathrm{~mm} \mathrm{x}$ $800 \mathrm{~mm}$ e $2 \mathrm{~mm}$ de espessura, houve a necessidade de confeccionar uma membrana cilíndrica para o teste, a partir de um rolo com dimensões superiores à dimensão requerida, para que não houvesse mais de um ponto de emenda. No local de colagem, que corresponde a uma geratriz do 
corpo de prova, foi deixada uma faixa de 100 mm em cada extremidade, formando uma área de sobreposição para a emenda. Estas áreas de emenda foram lixadas e limpas antes da aplicação da cola, para permitir melhor aderência. A secagem foi realizada com a membrana sendo mantida em um molde plástico com a mesma largura do corpo de prova, até a aderência entre as partes (Figura $3 b$ ).
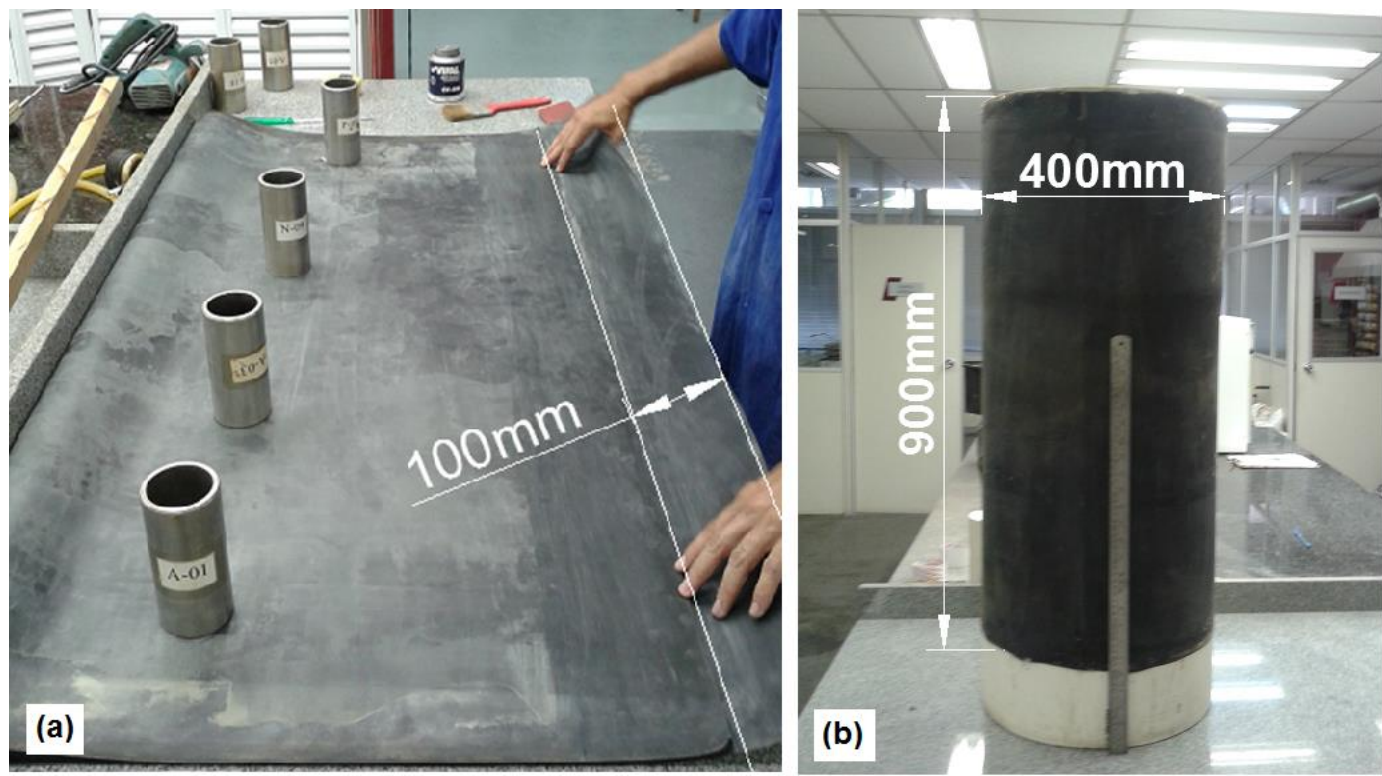

Figura 3 - (a) Detalhe da colagem da membrana e (b) membrana mantida no molde plástico para a secagem

\section{PREPARAÇÃO DO CORPO DE PROVA}

Para a realização do ensaio utilizou-se material oriundo do pátio de armazenamento de lastro da Vale S.A., localizado em Cariacica (ES), que fornece agregados para a Estrada de Ferro Vitória-Minas. Já em laboratório, a amostra foi homogeneizada e quarteada, conforme descrito na ASTM C702 (2003). A caracterização granulométrica indicou que o diâmetro máximo das partículas era $50 \mathrm{~mm}$, com a curva de distribui- ção de agregados sendo enquadrada na faixa de Graduação n ${ }^{\circ} 24$ do Manual da AREMA (2009), reproduzida na Figura 4. Neste caso, como o corpo de prova de $400 \mathrm{~mm}$ de diâmetro por 800 $\mathrm{mm}$ de altura permite que sejam realizados ensaios em partículas com diâmetro máximo de até $63 \mathrm{~mm}$, com uma relação de $\mathrm{D} / \mathrm{d}_{\text {máx }}$ de 6,35 , esta proporção se mostrou adequada, com base no que foi citado anteriormente.

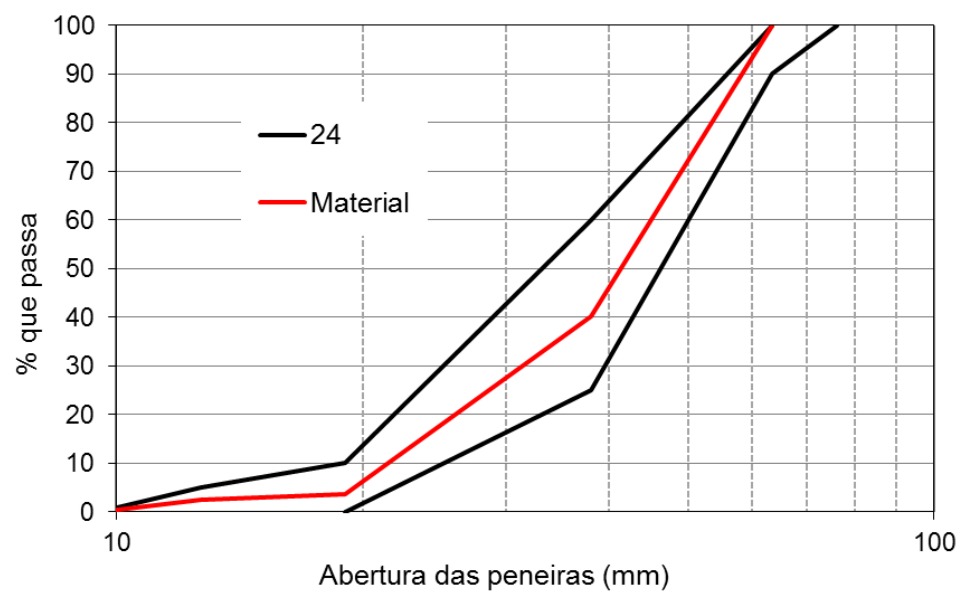

Figura 5 - Distribuição granulométrica do material utilizado em comparação com a faixa 24 AREMA (2009)

Os agregados foram divididos e homogeneizados em quatro porções, com massas iguais, de forma a ser executado um corpo de prova em quatro camadas, e assim minimizar uma possível segregação do material e conferir maior homogeneidade ao corpo de prova. Após a 
homogeneização, cada foi depositada no interior do molde e, posteriormente, vibrada a uma frequência de $20 \mathrm{~Hz}$ durante $40 \mathrm{~s}$. Ao final, atingiuse uma massa específica de $1,71 \mathrm{~g} / \mathrm{cm}^{3}$, com uma massa total da amostra de $172,1 \mathrm{~kg}$, obtendo-se um estado de compactação e rearranjo das partículas similar ao de campo (Selig; Waters, 1994; Indraratna et al. 2011). Após a compactação das quatro camadas, o corpo de prova teve sua superfície regularizada para a colocação do top cap. Em seguida, as extremidades do corpo de prova foram vedadas com silicone, tanto na base como no topo.

Após vibração e vedação do corpo de prova, todo o aparato foi transportado cuidadosamente pela grua até o atuador, visto que o po- sicionamento do corpo de prova na bancada é um processo crítico, devido à elevada massa do conjunto. Por fim, foram colocados ainda dois anéis de borracha, tanto na base como no topo, para impedir a entrada de ar entre a membrana e o corpo de prova. Em seguida, foi feita a aplicação de vácuo para o confinamento, ressaltandose que tanto a base como o topo possuem válvulas que permitem o controle de saída de ar, garantindo maior segurança ao sistema na ocorrência de algum problema de falta de confinamento. A Figura 5 ilustra algumas etapas de preparação do corpo de prova que foram descritas. Ao final deste processo, o corpo de prova já se encontrava preparado para a aplicação de carga.
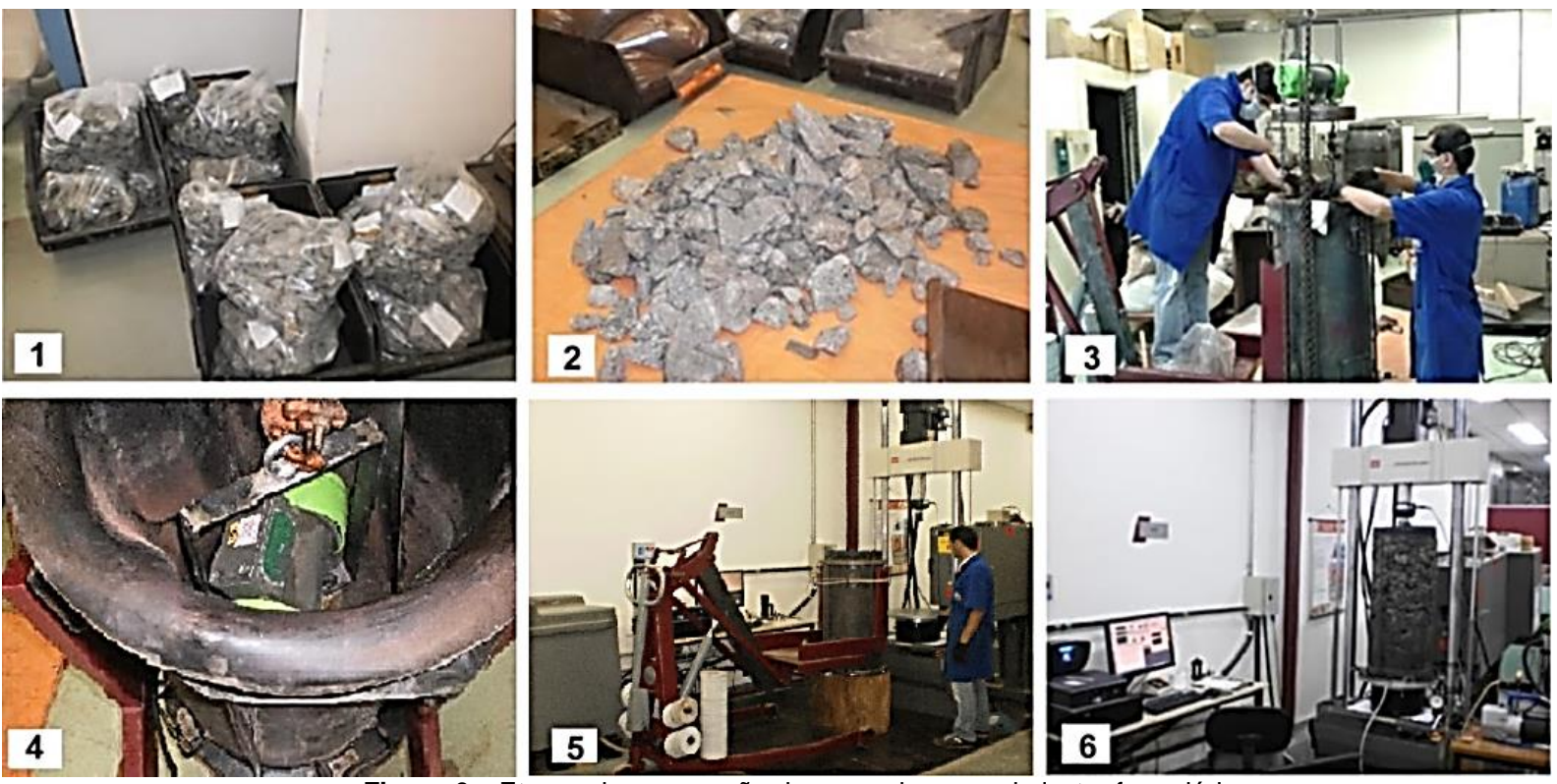

Figura 6 - Etapas de preparação do corpo de prova de lastro ferroviário

\section{TESTE CÍCLICO}

O cálculo da tensão a ser empregada nos ensaios seguiu os padrões citados por Talbot (1920) e Selig e Waters (1994), que se fundamentam em soluções de uma viga com apoiocontínuo e elástico em análises de Winkler
(1867) apud Kerr (1977). Os parâmetros adotados para o cálculo são apresentados na Tabela 2, cujos valores de referência foram obtidos na literatura (Alves; Sinay, 2005; Stewart, 1986; Esveld, 2001).

Tabela 2 - Valores de referência adotados para o cálculo das tensões atuantes

\begin{tabular}{ll}
\hline Parâmetro & Valor de Referência \\
\hline Espaçamento entre dormentes & $0,6 \mathrm{~m}$ \\
Módulo de via $(\mathbf{u})$ & $40 \mathrm{MPa}$ \\
Carga por eixo $\left(\mathbf{Q}_{\mathbf{0}}\right)$ & Variável \\
Fator de incremento dinâmico (FID) & 1,0 \\
Momento de inércia dos trilhos (I) & $2,04 \times 10^{-5} \mathrm{~m}^{4}$ \\
\hline Comprimento do dormente & $2,0 \mathrm{~m}$ \\
\hline Largura do dormente & $0,24 \mathrm{~m}$ \\
\hline
\end{tabular}


No cálculo foram consideradas diferentes cargas por eixo, representando ferrovias que transportam 15, 22, 27,5 e 32,5 toneladas por eixo (valores usualmente verificados no Brasil). Assim, foram obtidas as tensões abaixo dos dormentes (abaixo das áreas que suportam os trilhos), correspondentes à tensão principal $\left(\sigma_{1}\right)$ que atua na direção vertical da via permanente (sendo que se for subtraída a tensão confinante tem-se a tensão-desvio - $\sigma_{\mathrm{d}}$ ) com valores de 195 , 260,325 e $390 \mathrm{kPa}$ para cada tipo de ferrovia, supracitada, respectivamente.

A forma de carga cíclica típica a ser aplicada no ensaio triaxial é apresentada na Figura 7 , segundo o modelo usado por Indraratna et al. (2010a). A tensão mínima qmín cíclica foi mantida em $45 \mathrm{kPa}$, a qual representa o estado da via sem a passagem do trem, consistindo assim no peso da superestrutura. A quantidade de ciclos foi determinada a partir de Indraratna e Salim (2005) e Selig e Waters (1994), que descrevem que as maiores deformações acontecem logo nos primeiros ciclos de carregamento, em torno de 20.000 ciclos. Foram realizadas quatro combinações de tensões, iniciando-se pelo menor valor escolhido de $\sigma_{\mathrm{d}}$ até o maior, pois desta forma seria possível ensaiar diferentes condições sem a necessidade de moldar vários corpos de prova (Selig; Waters, 1994).

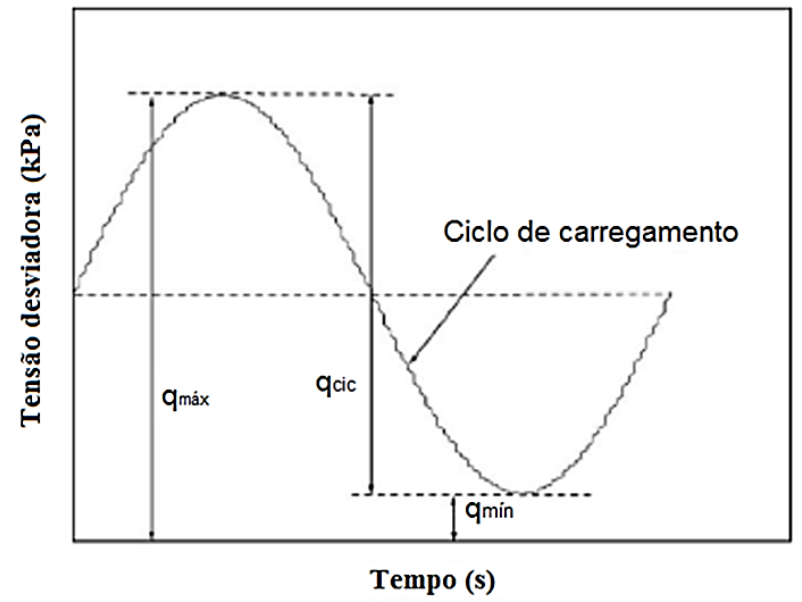

Figura 7 - Ciclo de carregamento típico utilizado nos ensaios triaxiais (Indraratna et al., 2010a)

Além da tensão vertical, também foi preciso determinar a tensão de confinamento, que influencia de maneira expressiva o comportamento mecânico do lastro, por este se tratar de um material granular. Procurou-se determinar a pressão confinante como sendo um valor coerente com a bibliografia, como o que foi obtido no estudo de campo realizado por Indraratna et al. (2010b), por exemplo. Na verdade, a estimativa das tensões de confinamento que atuam na camada de lastro é difícil de ser conhecida e, da mesma forma, simulada nos ensaios, devido ao comportamento geostático do material e à ação dinâmica causada pelos trens. Sendo assim, adotou-se uma tensão de confinamento de $65 \mathrm{kPa}$ (que simularia o caso de uma ferrovia com transporte de 27,5 toneladas por eixo), estando tal valor de acordo com relatos de Alva-Hurtado e Selig (1981), Indraratna e Salim (2005) e Indraratna et al. (2010b).

Com o objetivo de compreender o comportamento mecânico do lastro com confiabilidade, adotou-se uma quantidade de 20.000 ciclos para as relações de $\sigma_{1} / \sigma_{3}=3$ e 4 , onde os níveis de tensão são baixos e as deformações estabilizam-se rapidamente, e de 125.000 ciclos quando para $\sigma_{1} / \sigma_{3}=5$ e 6 , totalizando 290.000 ciclos de carregamento. $\mathrm{O}$ aspecto do corpo de prova durante o ensaio triaxial pode ser observado na Figura 7. No detalhe da figura é possível perceber que, com a aplicação do vácuo, a membrana de $2 \mathrm{~mm}$ de espessura envolve bem os agregados, garantindo o estado de tensões desejado. O equipamento de aplicação de vácuo mostrou-se confiável para a realização do teste, pois durante a ciclagem não houve queda na tensão de confinamento (fato este que poderia comprometer o ensaio, devido à forte influência desta tensão na resistência do material).

\section{RESULTADOS}

Para os níveis de tensão escolhidos, a evolução da deformação permanente ao longo do carregamento e a variação do módulo de resiliência podem ser vistos na Figura 8. Nas quatro combinações de tensões observa-se que o material sempre mostrou estabilização de deformação plástica após os ciclos iniciais (Figura 8a). Esta dependência do histórico de tensões em que o incremento de deformação plástica diminui geralmente com o aumento de ciclos para uma razão de tensões específica foi também descrita por Lim (2004). Destaca-se que as deformações plásticas do lastro se mantêm estabilizadas abaixo desta relação de tensões, porém quando esta é aumentada para níveis nunca antes aplicados, a deformação plástica continua se acumulando até atingir um novo ponto de equilíbrio ou 
alcançar a ruptura (teoria do shakedown) (Indraratna et al. 2011).

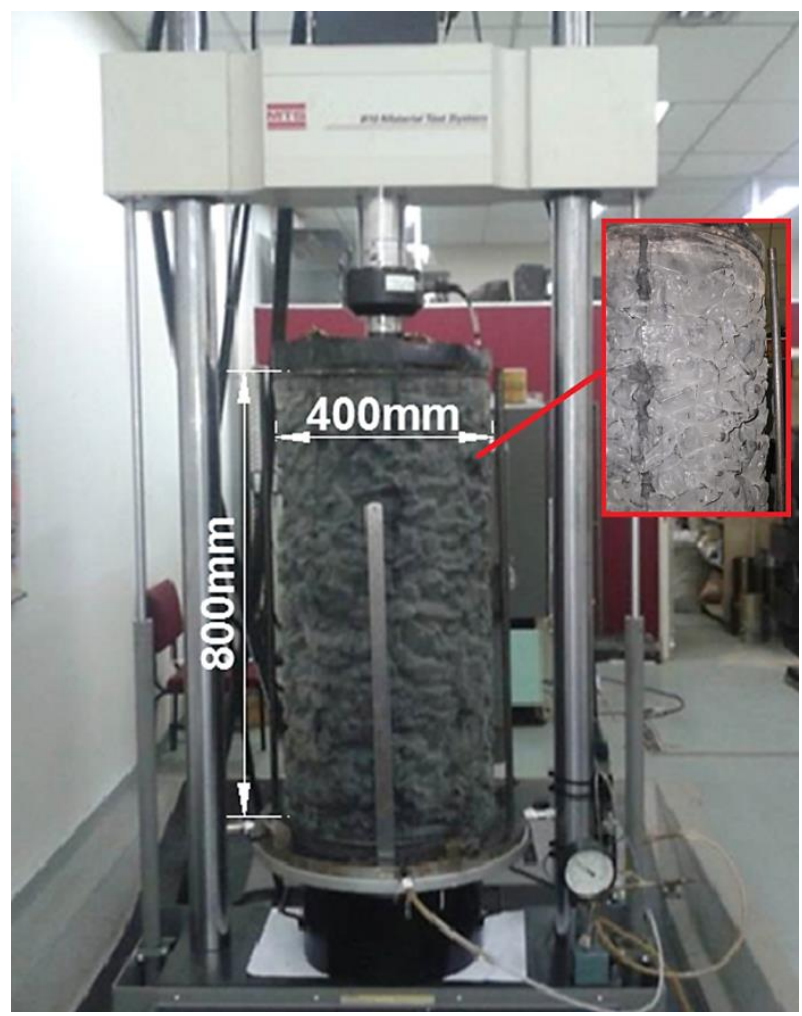

Figura 8 - Corpo de prova durante o ensaio triaxial

O efeito direto da alteração da taxa de deformação pode ser percebido pela variação do módulo de resiliência que, em geral, aumenta gradualmente com o número de aplicações de carga devido ao enrijecimento do material (Sevi, 2008; Indraratna; Salim, 2005), como observado na Figura 8b. Nos primeiros ciclos de cada combinação, os módulos de resiliência foram mais baixos, pois é justamente nesta fase que as deformações são maiores por conta da "reacomodação" das partículas. Após a fase de rearranjo, os valores de módulo de resiliência se elevaram ligeiramente até um nível de estabilização. Contudo, é importante destacar que este aumento no módulo de resiliência acontece até o ponto em que os finos, oriundos da quebra dos agregados, ainda não comandam o comportamento da matriz do material, pois a partir do momento em que este fenômeno acontece, os valores de módulo de resiliência caem. Este fato ocorre quando certa quantidade de finos, em torno de mais de $30 \%$ da massa, produzidos pela quebra e por contaminações diversas (solo do subleito, poeira do ambiente, material que cai dos vagões na via, etc); tal comportamento é percebido em campo e pode ser potencializado quando os finos entram em contato com água (considerando uma situação de má drenagem, por exemplo). Nestes casos, os finos agem como lubrificantes do contato agregado-agregado e promovem um maior deslocamento do conjunto sob carga, reduzindo o módulo de resiliência. Em contrapartida, os módulos de resiliência podem aumentar consideravelmente caso a quantidade de finos que colmata os vazios do lastro for expressiva e estes secarem, elevando também a tensão de sucção deste material. A variação de queda e aumento de módulo de resiliência do lastro é altamente indesejável, pois causa variações de cargas dinâmicas e desgastes irregulares nos trilhos por apresentar heterogeneidade de comportamento mecânico.

Ainda se observa na Figura 8b que a tensão desviadora influencia nos resultados de módulo de resiliência, visto que quanto maiores as tensões aplicadas maiores foram os valores de módulo de resiliência encontrados. A Figura 9 apresenta as curvas tensão-deformação do ensaio cíclico para a amostra de lastro nas quatro relações de tensão testadas. A captação de dados realizada pela célula de carga ocorreu em uma frequência superior a $110 \mathrm{~Hz}$, o que garantiu a completa representação das deformações ao longo de um ciclo de carregamento. Os dados apresentados na Figura 9 correspondem aos diferentes ciclos de carga escolhidos, para cada combinação de tensão. Pelos dados, percebe-se claramente o comportamento elastoplástico do material de lastro ferroviário, que se caracteriza pelo acúmulo gradual de deformação permanente a cada aplicação de carga, até a diminuição da taxa de deformação plástica à medida que o número de ciclos aplicados aumenta.

Outro fato importante evidenciado pela $\mathrm{Fi}$ gura 9 é a aplicação de carga realizada pelo atuador: durante todo o teste, o equipamento conseguiu aplicar com precisão as diferentes tensões solicitadas, no caso tanto a tensão residual ( $\left.\mathrm{q}_{\text {mín }}\right)$ quanto a tensão de pico ( $\left.\mathrm{q}_{\text {máx }}\right)$. Isto representa confiabilidade nos resultados, pois segundo Aursudkij (2007), ensaios com corpos de prova nestas dimensões, submetidos a altas frequências, apresentam dificuldades com relação à aplicação das tensões solicitadas. 


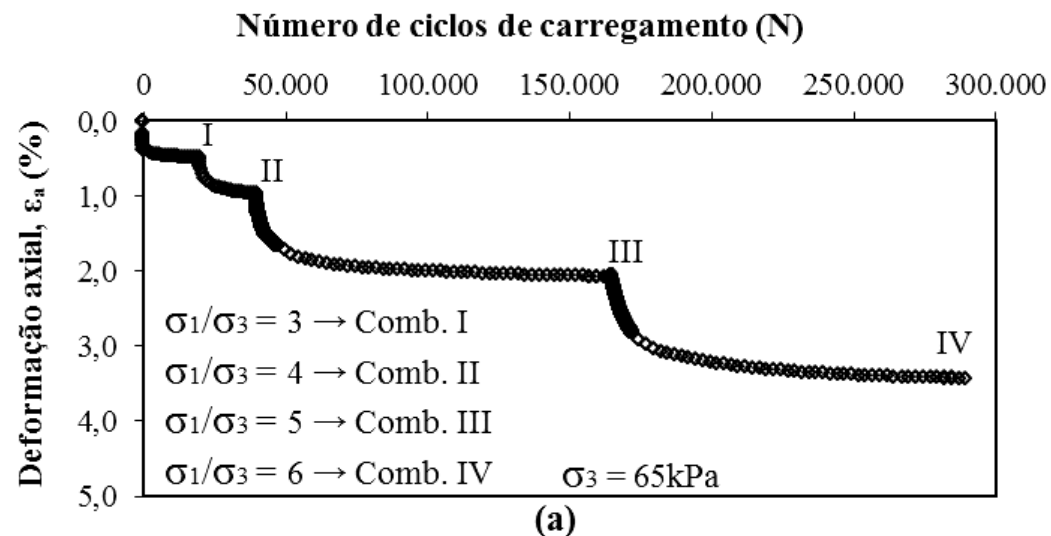

(a)

Número de ciclos de carregamento $(\mathbf{N})$

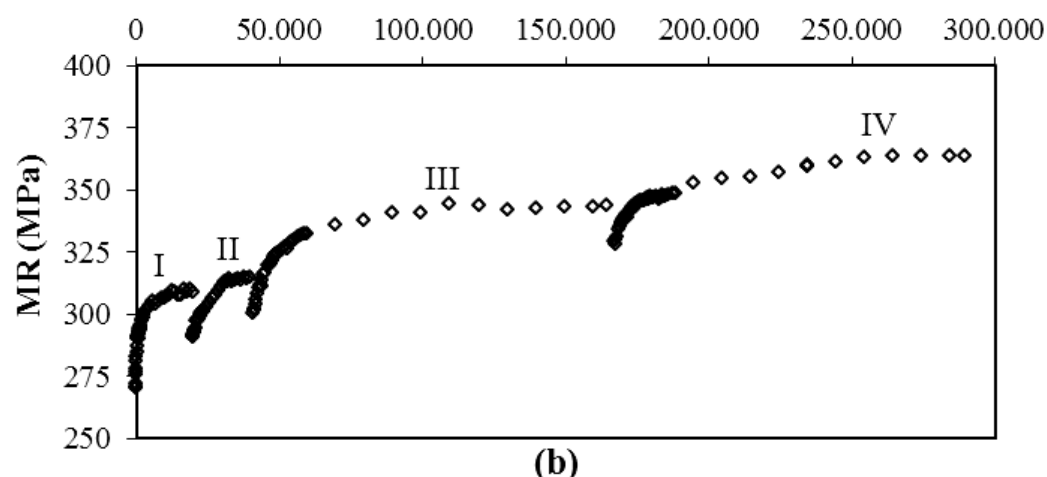

Figura 9 - (a) Deformação permanente axial; e (b) Módulo de resiliência ao longo de 290.000 ciclos
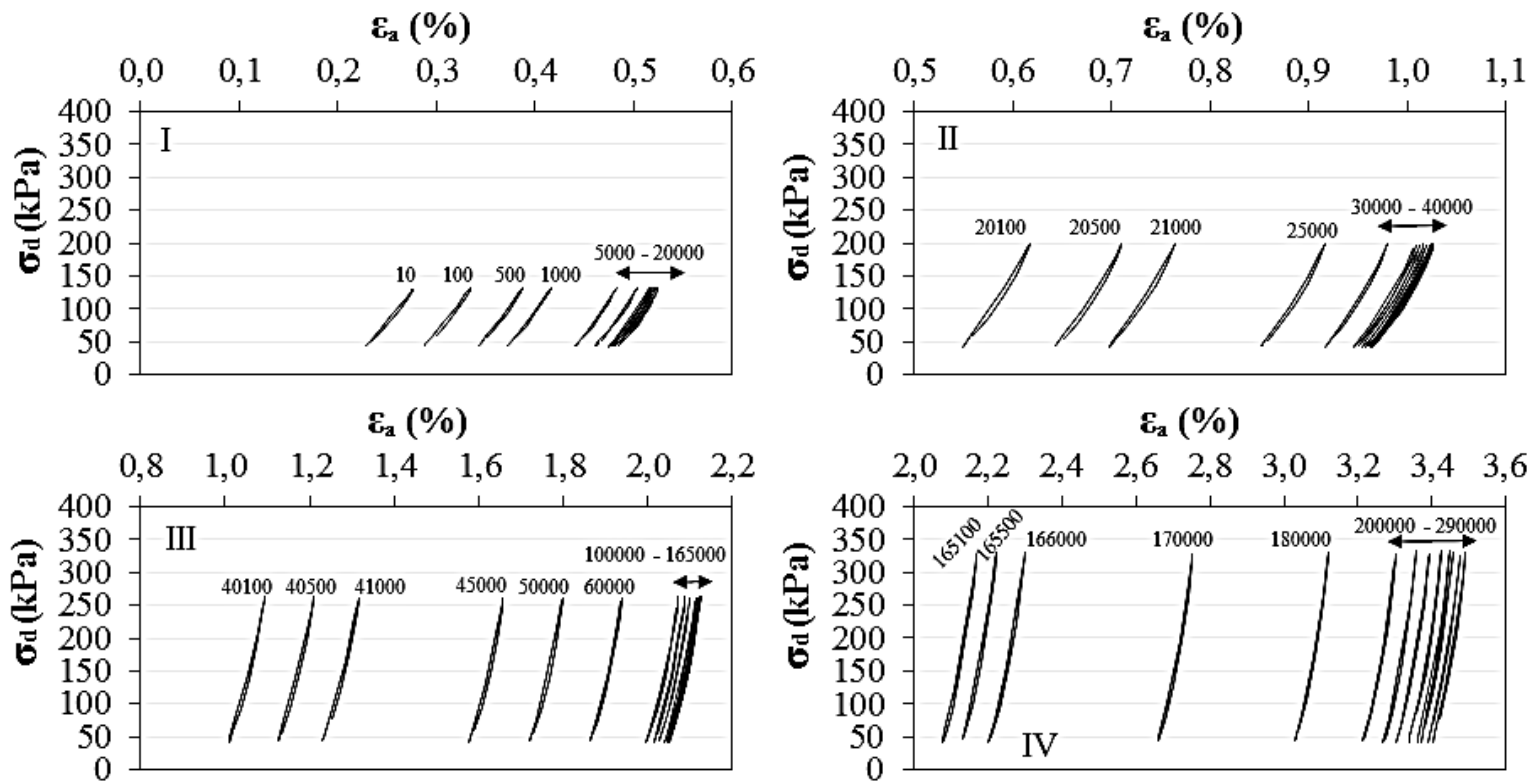

Figura 10 - Curvas tensão-deformação para várias combinações de carregamento

Após o ensaio, pôde-se observar a deterioração do material pela fragmentação das partículas, conforme ilustrado na Figura 10. Pela imagem, fica claro que o carregamento cíclico, além de causar o rearranjo das partículas (situa- ção já citada), também ocasionou quebra das mesmas, demonstrando que o nível de tensão escolhido e as condições de ensaio são adequados e caracterizam satisfatoriamente o comportamento típico de uma ferrovia. 

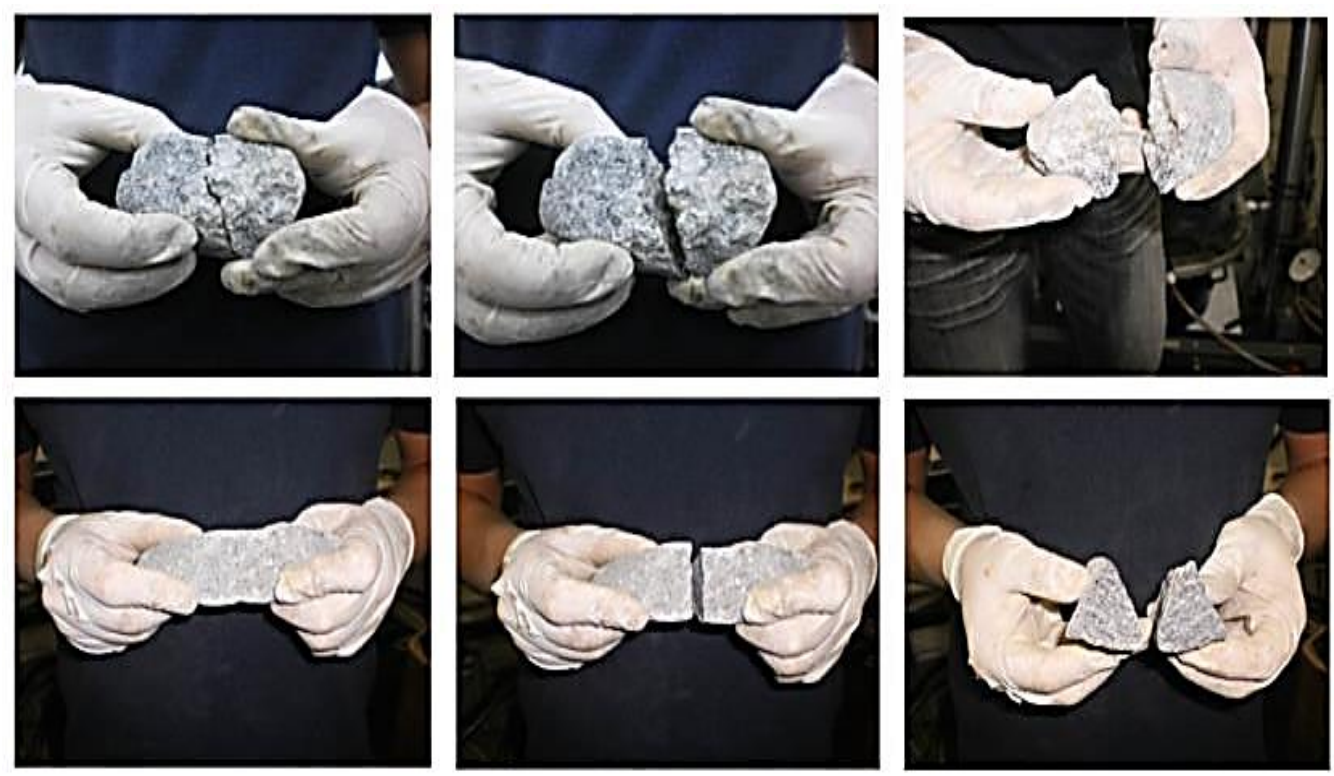

Figura 11 - Agregados deteriorados após ensaio mecânico em grande escala

\section{CONCLUSÕES}

Neste trabalho foi apresentado o desenvolvimento de um equipamento triaxial de grande escala para estudo de material de lastro ferroviário, capaz de testar amostras com tamanho máximo de partícula de até $63 \mathrm{~mm}$ com representatividade. Este equipamento é inédito no Brasil e apresenta uma nova alternativa em pesquisas de lastro de ferrovias.

O método de preparação do corpo de prova mostrou-se satisfatório, pois os resultados obtidos no ensaio estão de acordo com a bibliografia pesquisada. Os ensaios de grande escala mostram boas correlações com o comportamento mecânico da camada lastro. O nível de tensões escolhido mostrou-se satisfatório para simular a via permanente sob carregamento de trens de passageiros e até de carga, como pôde ser visto pela deterioração dos agregados ao final do teste. Este equipamento permite a análise de diferentes materiais e granulometrias para lastros empregados em ferrovias. Entretanto, entende-se que seria importante realizar uma maior quantidade de ensaios laboratoriais para proporcionar melhores correlações e simulação das condições de campo.

Pelos bons resultados demonstrados neste teste, o estudo deve se estender para a avaliação das características do lastro ferroviário em condições de carga variadas e a determinação a influência dos diversos parâmetros que afetam o comportamento deste material, a fim de contribuir para um projeto de vias férreas mais realistas, por exemplo. Com isto, pode-se minimizar a deformação e a degradação da camada de lastro, permitindo o nivelamento adequado da via por mais tempo e, consequentemente, a redução nos custos de manutenção.

\section{AGRADECIMENTOS}

Ao CNPq pelo apoio na forma de bolsa de mestrado do primeiro autor e à empresa Vale S.A. pela parceria em projeto de pesquisa.

\section{REFERÊNCIAS}

AASHTO T 292-91 - Interim method of test for resilient modulus of subgrade soils and untreated base/subbase materials. American Association of State Highway And Transportation Officials, Washington, D.C., 1991.

Alva-Hurtado, J. E., and Selig, E. T. Permanent strain behavior of railway ballast. Proceedings of $10^{\text {th }}$ International Conference on Soil Mechanics and Foundation Engineering. Pergamon Press:New York, 543-546, 1981.

Alva-Hurtado, J. E.; Mcmahon, D. R.; Stewart, H. E. Apparatus and techniques for static triaxial testing of ballast. Laboratory Shear Strength of Soil, ASTM STP 740, R.N. Yong and F.C. Townsend, EDS., American Society for Testing and Materials, 1981, pp.94-113.

Alves, G. K. A., Sinay, M. C. F. Os dormentes ferroviários, seu tratamentos e o meio ambiente. [2005]. Disponível em: <http://www.cbtu.gov.br/estudos/pesquisa>. Acesso em:13/06/2012.

Anderson, W. F.; Fair, P. Behavior of railroad ballast under monotonic and cyclic loading. Journal of Geotechnical and Geoenvironmental Engineering, Vol. 134, No. 3, 2008. DOI: 10.1061/(ASCE)1090-0241(2008)134:3(316)

AREMA: Manual for Railway Engineering. American Railway Engineering, 2009. 
ASTM C702 (2003) Standard Practice for Reducing Samples of Aggregate to Testing Size. American Society for Testing and Materials, ASTM.

ASTM D5311. Standard Test Method for load controlled cyclic triaxial strength of soil. American Society for Testing and Materials, 2004.

Aursudkij, B., Mcdowell, G. R., Collop, A. C. Cyclic Loading of Railway Ballast under Triaxial Condition in a railway test facility. In: Granular Matter, Vol. 11, No. 6, 2009, pp.391-401. DOI: 10.1007/s10035-009-0144-4

Aursudkij, B.: A Laboratory Study of Railway Ballast Behaviour under Traffic Loading and Tamping Maintenance. Thesis, The University of Nottingham, United Kingdom, 2007.

Bishop, A. W. and Green, G. E. (1965).The influence of end restraint on the compression strength of a cohesionless Soil. Geotechnique 15, pp. 243-266. DOI: 10.1680/geot.1965.15.3.243

Diyaljee, V. A.: Effects of stress history on ballast deformation. Journal of the Geotechnical Engineering, ASCE, Vol. 113, No. 8, 1987, pp. 909-914. DOI: 10.1061/(ASCE)07339410(1987)113:8(909)

Ebrahimi, A; Tinjum, J. M.; Edil, T. B. Large-Scale, cyclic triaxial testing of rail ballast. AREMA Annual Conference e Exposition, Orlando, 2010.

Esveld, C. Modern railway track. MRT Productions, 2001, $654 \mathrm{p}$.

Fortunato, E. Renovação de Plataformas Ferroviárias. Estudos Relativos à Capacidade de Carga. PhD Thesis. Porto: University of Porto. 2005.

Indraratna, B., Thakur, P., Vinod, J. Experimental and Numerical Study of Railway Ballast Behavior under Cyclic Loading. International Journal of Geomechanics, Vol. 10, No. 4, 2010a, pp 136-144. DOI: 10.1061/(ASCE)GM.19435622.0000055

Indraratna, B., Ionescu, D., Christie, D., Chowdhury, R. Compression and degradation of railway ballast under onedimensional loading. Australian Geomechanics, 1997, pp 48-61.

Indraratna, B., Nimbalkar, S., Christie, D., Rujikiatkamjorn, C., Vinod, J. Field assessment of the performance of a balasted rail track with and without geosynthetics. Journal of the Geotechnical and Geoenvironmental Engineering, Vol. 136, No. 7, 2010b, pp. 907-917. DOI: 10.1061/(ASCE)GT.19435606.0000312

Indraratna, B., Salim, W. Mechanics of ballasted rail tracks: a geotechnical perspective. Taylor \& Francis Group plc. Londres, 2005, $248 \mathrm{pp}$

Indraratna, B.; Ionescu, D.; Christie. H. D. Shear Behavior of Railway ballast based on large-scale triaxial tests. Journal of Geotechnical and Geoenvironmental Engineering, Vol. 124, No. 5 , 1998, p. 439-449. DOI: 10.1061/(ASCE)10900241(1998)124:5(439)

Indraratna, B.; Salim, W.; Rujikiatkamjorn, C. In: Advanced rail geotechnology ballasted track. Taylor \& Francis Group, London, UK, 2011.
Kerr, A. D. On the Stress Analysis of rails and Ties. Bulletin

659, in: Proceedings of the American Railway Engineering Association, Vol. 78, 1977, pp. 19-43.

Lackenby, J.; Indraratna, B.; Mcdowell, G.; Christie, D. Effect of confining pressure on ballast degradation and under cyclic triaxial loading. In: Géotechnique 57, No. 6, 2007, pp. 527-536. DOI: $10.1680 /$ geot.2007.57.6.527

Lim, W. L. Mechanics of Railway Ballast Behaviour. PhD Thesis. University of Nottingham. 2004, 216 p.

Malysz, R. Desenvolvimento de um equipamento triaxial de grande porte para a avaliação de agregados utilizados como camada de pavimentos. 2009. 350p. Tese (Doutorado) Programa de Pós-graduação em Engenharia Civil, Universidade Federal do Rio Grande do Sul. Porto Alegre, 2009.

Merheb, A. Análise mecânica do lastro ferroviário por meio de ensaios triaxiais cíclicos. Dissertação de Mestrado, POLI/USP, São Paulo, Brasil, 2014. 148 p.

Nalsund, R. Effect of grading on degradation of crushed-rock Railway Ballastand on Permanent Axial Deformation. Transportation Research Record, Washington, D. C., No. 2154, 2010, p.149-155.

Raymond, G. P., and Diyaljee, V. A. Railroad ballast sizing and grading. Journal of Geotechnical Engineering, ASCE, 105(5), 676-681, 1979

Sekine, E.; Kono, A.; Kito, A. Strength and deformation characteristics of railroad ballast in ballast particle abrasion process, 2005.

Selig, E. T., Waters, J. M. Track geotechnology and substructures Management. Thomas Telford Services Ltd., Londres, 1994. 446 pp.

Sevi, A. S. Physical modelling of railroad ballast using parallel gradation scaling technique within the cyclical triaxial framework. Tese de doutorado.Missouri University of science and Technology. 2008. 137p.

Sevi, A. S.; Ge, L.; Take, W. A. A large-scale triaxial apparatus for prototyperailroad ballast testing. Geotechnical testing journal, Vol. 32, No. 4, 2009, pp.1- 8 .

Skoglund, K. A., 2002, A study of Some Factors in Mechanistic Railway Track Design, Ph.D. dissertation, Norwegian University of Science and Technology.

Stewart, H.E.: Permanent strains from cyclic variable amplitude loadings. J. of Geotechnical Engineering, ASCE, Vol. 112, No. 6, 1986, pp. 646-660. DOI: 10.1061/(ASCE)07339410(1986)112:6(646)

Suiker, A. S. J.; Selig, E. T.; Frenkel, R. Static and cyclic triaxial testing of ballast and subballast. Journal of Geotechnical and Geoenvironmental Engineering, Vol. 131, No. 6, 2005. DOI: 10.1061/(ASCE)1090-0241(2005)131:6(771)

Talbot, A. N. Second progress report of the Special Committee on Stresses in Railroad Track. Proceedings of the AREA, Vol. 21, 1920, pp. 645-814. 